\title{
A FILOSOFIA DA CIÊNCIA E A FILOSOFIA DA QUÍMICA: UMA PERSPECTIVA CONTEMPORÂNEA
}

\author{
Lisandro Bacelar da Silva ${ }^{1}$ \\ Uarison Rodrigues Barreto ${ }^{2}$ \\ Nélson Rui Ribas Bejarano ${ }^{3}$ \\ Marcos A. P. Ribeiro ${ }^{4}$
}

RESUMO: Este trabalho tem por objetivo identificar e caracterizar o atual estado da relação entre a filosofia da ciência contemporânea e a filosofia da química. Trata-se de uma abordagem qualitativa, constituindo-se de um estudo exploratório do tipo estado da arte. Inicialmente, refletimos sobre algumas razões que culminaram no histórico negligenciamento da filosofia da química pela filosofia da ciência. Em seguida, a partir de uma breve caracterização desta relação, argumentamos que algumas dimensões, como o complexo estilo de pensamento e fazer químico, devem ser apropriadas pela filosofia da ciência. Por fim, defendemos a necessidade de maior inserção das filosofias da química e da ciência, no âmbito da formação de professores de química. 
PALAVRAS-CHAVE: filosofia da ciência; filosofia da química; negligenciamento; especificidade do pensamento químico; educação química.

ABSTRACT: This paper aims to identify and characterize the current state of the relationship between the contemporary philosophy of science and philosophy of chemistry. This is a qualitative approach, becoming an exploratory study of the type state of the art. Initially, we reflect on some reasons that culminated in the historic neglect of the philosophy of chemistry from the philosophy of science. Then, from a brief description of this relationship, we argue that some dimensions as the complex style of thinking and doing chemical should be appropriate for the philosophy of science. Finally, we advocate the need for greater integration of chemical philosophy and philosophy of science in the training of chemistry teachers.

KEYWORDS: philosophy of science; philosophy of chemistry; neglect; specificity of chemical thinking; chemical education. 


\section{O PROBLEMA DO NEGLIGENCIAMENTO}

Compreendemos, assim como Ribeiro (2014), que a filosofia da ciência tem historicamente privilegiado esquemas epistemológicos, essencialmente, físicomatemáticos em detrimento de outros modelos, como o químico (VAN BRAKEL, 2000; HOFFMANN, 2007). Nesse sentido:

Quando olhamos as grandes formulações da filosofia e da epistemologia, desde Descartes a Kant, de Augusto Comte a Pierre Duhem, de Karl Popper a Paul Feyerabend, constatamos que elas estão baseadas numa concepção física do mundo (RIBEIRO, 2014, p.38-39).

Para Labarca (2005), mesmo na atualidade, quando a filosofia da ciência se dividiu em diversas subdisciplinas, dedicando-se a analisar os problemas das ciências específicas, a filosofia da química estava, até bem pouco tempo, ausente. Mas, "por que a ciência mais produtiva e, portanto, a grande maioria da ciência produzida no século XX foi negligenciada" (RIBEIRO, 2014)? Conforme salienta este autor, filósofos da Química, como Bensaude-Vincent (2010), Schummer (2006), Sjostrom (2006), Scerri (2007) e Van Brakel (1999), apontam explicações para o negligenciamento, entendendo que a elaboração do 
conhecimento químico, sua natureza e sua peculiar forma de explicar os fenômenos da natureza, foram preteridas por parte da hegemônica Filosofia da Ciência.

Uma das razões para o negligenciamento remete à proximidade da química com a tecnologia - associada a suas raízes históricas no pragmatismo e no intervencionismo, tornando difícil a visualização de uma separação entre ciência acadêmica e industrial - o que explicaria, para Sjostrom (2006), Schummer (2006) e Van Brakel (1999), a escassa tendência da química a construir teorias e modelos universais tão valorizados pela Filosofia da Ciência. Conforme sublinha Ribeiro, para Van Brakel, "a física pergunta-se sobre o que é o universo, $\{. .$.$\} ao passo que$ a química, aparentemente, não tem uma pergunta fundante que remeta a grandes inquirições filosóficas" (2014, p.73).

Caldin (1961), escrevendo sobre a natureza das leis e teorias químicas, destaca que estas não têm o mesmo sentido que as leis e teorias da física: as leis químicas são peculiares, não têm o sentido de universalidade; funcionam mais no sentido de regras para a ação do que como leis gerais para explicação. Um exemplo seria a lei periódica dos elementos, "a ocorrência periódica dos elementos, após certo intervalo, é aproximada" (SCERRI, 2000b, p.523). Esta distinção sugere que as leis da natureza podem ter diferenças ao tratar de cada disciplina científica específica. Em "The Preference of Models over Laws of Nature in 
Chemistry", Schummer (2014) defende o uso dos modelos frente às leis da natureza. Nesse sentido, argumenta que a química, como o epítome original da ciência experimental (ou Baconiana), parte do pluralismo metodológico que entre uma variedade de modelos, a escolha é a partir de razões pragmáticas, tendo esse procedimento preferência sobre leis numa perspectiva universal, como na física matemática. $\mathrm{Na}$ atualidade, Vihalemm $(2003,2011)$ tem sido um dos filósofos que mais tem explorado o contexto da natureza das leis químicas.

Os químicos estariam mais inclinados a serem instrumentalistas ou pragmatistas do que cientistas realistas à procura de leis universais, e a filosofia, em contrapartida, desde o final do século XIX, tem se interessado fundamentalmente pela concepção de teoria enquanto busca de unidade, não havendo, na química, nenhuma teoria em desenvolvimento que pudesse competir com a física (VAN BRAKEL, 1999). Em uma linha de raciocínio próxima a de Caldin e Van Brakel, Bensaude-Vincent (2010), entendendo o modo de elaboração do conhecimento químico no âmbito do saber-fazer, próprio de um realismo operativo, pouco habituado a tecer macro-narrativas sobre o universo, compreende que tal especificidade epistemológica contribuiu bastante para o afastamento da química do centro do debate da filosofia da ciência, visto que esta, para a filósofa e historiadora da química, tendeu a 
conceber um modelo de ciência baseado em uma ciência ideal e teórica, afastado, por conseguinte, do mundo da práxis (RIBEIRO, 2014).

Ribeiro, refletindo sobre a primazia que a física alcançou no âmago da filosofia da ciência, sublinha:
A física teórica, com as questões da mecânica quântica e da relatividade teve muita influência na profissionalização da filosofia da ciência durante os anos 30 e na obra de importantes filósofos no desenvolvimento da filosofia da ciência, o que levou a considerar a filosofia da física como a própria filosofia da ciência (RIBEIRO, 2014, p.74).

Até pelo menos a década de 1960, as questões centrais em filosofia da ciência remetiam quase exclusivamente à teoria da ciência, sua estrutura lógica e sintática. Parece que o legado do positivismo lógico e o domínio da Física em análises filosóficas ainda persistem, não obstante o fato de que a ênfase nas análises lógicas de teorias científicas tenha sido desafiada por filósofos, como Popper e Kuhn (ADÚRIZ-BRAVO, 2001; VAN BRAKEL, 1999). Desse modo, a física, mais envolvida com programas operados por modelos matemáticos, está repleta de sucessos preditivos, diferentemente da química, que opera com modelos qualitativos e classificatórios (RIBEIRO, 2014). 
Outra relevante explicação para este negligenciamento, associada à forte influência da Física, pode ser obtida a partir da análise do problema do reducionismo, conforme concebem Scerri (2007) e Ribeiro (2014): se a química pode ser reduzida a física de forma completa, se os fenômenos químicos podem ser explicados por conceitos da física, em particular, os da Mecânica Quântica.

Lombardi e Castagnino (2010) mostram que uma das dificuldades do reducionismo da química à física está em aceitar o reducionismo a uma ontologia quântica que não se sabe ainda o que significa. Sobre a mesma temática, Scerri (2007) comenta que a discussão sobre a redução está diretamente relacionada com a possibilidade de que os conceitos em química podem, ou não, ser reduzidos à Física (relação ambígua a que o autor se refere) mediada pela Mecânica Quântica, o que pode trazer implicações profundas para o ensino de Química. Por exemplo, um dos possíveis impactos sobre o ensino de química que teríamos é a ênfase demasiada em aspectos quantitativos em detrimento de aspectos qualitativos. Scerri, um crítico da redução, sugere como resposta a questão da redução: "Sim e Não". De sua visão, podemos extrair duas possibilidades: a redução parcial da química à física; a química reduzida e não reduzida ao mesmo tempo. 
O reducionismo destaca que a única explicação científica legítima encontra-se no nível mais micro, entendendo ser este o mais fundamental; assim, os outros níveis são apenas epifenômenos de uma realidade básica e fundamental que é propriamente a realidade física, recebendo o nome também de fisicalismo (SCERRI, 2007). Logo, na medida em que a química era vista como reduzível ontologicamente à mecânica quântica, obscureciam-se problemas filosóficos maiores. Dado o histórico interesse pelas leis e teorias unificadas, a hegemônica filosofia da ciência considerou a química como parte não muito importante da física, mesmo uma física aplicada, tão somente preocupada com aspectos fenomenológicos, uma prática cujo nível explicativo estava no domínio da física. Quando, no século XX, a mecânica quântica oferece uma explicação parcial para a ligação química, esta tensão se acirra (RIBEIRO, 2014). Cremos que o caráter redutivo atenta contra a autonomia da química, uma vez que, coloca a química numa posição subordinada à física. A despeito disso, nossa posição se aproxima da concepção de Labarca e Lombardi (2005), os quais defendem a autonomia da química quanto aos aspectos epistemológicos e ontológicos.

Ainda destacamos que a filosofia da química na França, entre 1900-1960, não foi negligenciada. Mas, por que razão? Para Van Brakel (2012), é porque a história da ciência é necessária para a compreensão da ciência. Isso 
estava presente principalmente entre Duhem, Meyerson, Bachelard e outros. Critica Kant, pois não pode haver ciência pura. Bensaude-Vincent (2005) considera que a tradição francesa foi a única corrente filosófica que não negligenciou a química, chegando inclusive a influenciar a filosofia bachelardiana, ao instaurar o entendimento do real como um processo de objetivação a partir da categoria fenomenotecnia (RIBEIRO, 2014).

\section{A FILOSOFIA DA QUÍMICA: UM CAMPO EMERGENTE DA FILOSOFIA DA CIÊNCIA}

Em um contexto de superação dos limites disciplinares de um campo essencialmente interdisciplinar e fragmentado, nasce, em 1997, a filosofia da química (RIBEIRO, 2014; SCHUMMER, 2006) como uma subdisciplina da Filosofia das Ciências e é estabelecida a International Society for the Philosophy of Chemistry. Não obstante, salientamos que, desde o final da década de 1980, grupos constituídos por químicos, filósofos e historiadores trabalhavam regularmente nos EUA e em diversos países europeus como parte de um processo de afirmação da Filosofia da Química como campo legítimo de investigação filosófica (VAN BRAKEL, 1999; LABARCA; BEJARANO; EICHLER, 2013; SILVA, 2015). 
Desde o início da década de 1990, que a filosofia da química examina a natureza específica do conhecimento químico, tendo como marco de sua institucionalização o ano de 1994 (RIBEIRO, 2014). Algumas implicações desse domínio em educação química têm sido investigadas, embora o território de investigação nesta área continua pouco explorado (RIBEIRO, 2014; SCHUMMER, 2006). Acredita-se que os tópicos discutidos e debatidos no campo disciplinar da Filosofia da Química podem ser utilizados na formação de professores, a fim de que os docentes desenvolvam uma concepção epistemológica mais profunda da Química (LABARCA; BEJARANO; EICHLER, 2013; RIBEIRO, 2014).

Uma tentativa importante de integração da filosofia da química no currículo inicial de professores de química é defendida por Ribeiro (2014). Apesar de ser um campo amplo, aberto, ainda muito pouco explorado e de difícil penetração (SCHUMMER, 2006), tal integração é possível com base na aproximação entre filosofia, química e currículo por meio do debate do campo disciplinar da filosofia da química (RIBEIRO, 2014; VAN BRAKEL, 1999, 2000). Entretanto, a filosofia da química ainda é uma disciplina essencialmente americana e europeia. A revista Hyle (International Journal for Philosophy of Chemistry), desde 1995, editada por Joachim Shummer, tem grande contribuição da Alemanha, França e Itália, países que têm fracas contribuições na revista Foundations of Chemistry. A Foundations of Chemistry, 
desde 1999, editada por Eric Scerri tem grande contribuição dos EUA e do Reino Unido.

O debate da filosofia da química na América Latina já é forte em países, como a Argentina, Colômbia, México, Venezuela, Uruguai. Na Argentina, temos a presença de autores importantes da filosofia da química: a professora Olímpia Lombardi (Conicet), Martín Labarca (Conicet) e Agustín Adúriz-Bravo (UBA) da História e Filosofia da Ciência. No Brasil, apesar de ter a segunda maior comunidade de químicos no mundo, a filosofia da química apresenta pouca representação. Encontramos entre os autores, Rosária Justi (UFMG), Marcos Ribeiro (UESB), Jackson Gois da Silva (UNESP), Nelson Rui Ribas Bejarano (UFBA) e Waldmir Araújo-Neto (UFRJ).

Mesmo hoje, com o campo da Filosofia da Química plenamente estabelecido as discussões em torno do denominado "problema da redução" - associadas a considerações que remetem a um entendimento da Física como ciência "fundamental", cujo conhecimento representaria a essência da realidade, restando à Química o papel de disciplina meramente "fenomenológica" na medida em que apenas descreveria a realidade tal como se apresenta - constituem a temática de maior maturidade nesta área. A despeito disso, problemas ligados ao conceito de estrutura molecular, orbital atômico, ligação química e aos fundamentos da tabela periódica vêm ganhando cada 
vez maior relevância no interior da Filosofia da Química (LABARCA; BEJARANO; EICHLER, 2013; SILVA, 2015).

\section{O COMPLEXO ESTILO DE PENSAMENTO E FAZER QUÍMICO: UM CAMPO RICO A SER APROPRIADO PELA FILOSOFIA DA CIÊNCIA}

Pensamos que algumas dimensões da filosofia da química, notadamente o complexo estilo de pensamento químico, devem ser apropriadas pela filosofia da ciência. Nesse intuito, a dimensão multicontextual (RIBEIRO, 2014; SCHUMMER, 2006) assumida pela química contemporânea, pode (e deve) ser um objeto de destaque na reflexão epistemológica da ciência, a qual parece valorizar demasiadamente o contexto da justificativa dentre outros tão relevantes como os de descoberta, aplicação e educação (RIBEIRO, 2014). Enquanto os filósofos da física exploram o contexto de justificativa, os filósofos da química exploram o contexto de descoberta, pois entendem que os químicos, ao mesmo tempo, que lidam com estruturas ordenadas como moléculas e cristais, lidam bastante com criações, principalmente, nas sínteses químicas (SCHUMMER, 2004).

Deste modo, a filosofia da química centra suas análises na afinidade, coesão, arquitetura, atração, harmonia e estética química (BENFEY, 2000; SCHUMMER, 
2006; HOFFMAN, 2007). Além de criativa, a química é também uma ciência amparada por um contexto de aplicação, em que os químicos, bastante próximos do experimento, realizam um trabalho simultaneamente artesanal e acadêmico. Tal dimensão prática possibilita aos conceitos químicos um enorme ganho de significado (ADÚRIZ- BRAVO, 2003; KOVAC, 2002; RIBEIRO, 2014).

$\mathrm{O}$ contexto da educação é bastante sublinhado por Seco, Alvarez e Sales (2008), os quais consideram que o desenvolvimento da química origina-se de uma atitude pedagógica; um pensamento semelhante ao de Bachelard, no qual defende que a química progride por ideias sistemáticas. A tese da centralidade do contexto educativo no crescimento da química é também defendida por Izquierdo-Aymerich (2012). Para esta autora, a química tem origens didáticas, tendo sido criada para ser ensinada.

De modo semelhante, o fazer químico efetiva-se como um campo rico para atentas explorações da filosofia da ciência (BENSAUDE-VINCENT, 2008), na medida em que concebendo a teoria como narrativa experimental (HOFFMANN, 1993, 2007), constitui-se numa prática que pouco se destina a desvelar um real subjacente a macrofenômenos ou testar hipóteses teóricas (CALDIN, 1961). Amparada por uma específica análise da história da Química, Bensaude-Vincent (2008) considera o debate entre positivismo é realismo bastante inadequado para uma 
compreensão do estilo de pensamento químico, uma vez que os químicos não objetivam representar a estrutura da realidade material (RIBEIRO, 2014), mas identificar disposições específicas para suas operações. Assim, para esta pesquisadora francesa, negações do estatuto ontológico de determinadas entidades químicas necessariamente não inserem a Química nos limites de uma ontologia positivista.

Afim de bem caracterizar todo este complexo estilo de pensamento e fazer químico, Bensaude-Vincent (2008) entende ser a categoria realismo operativo bastante apropriado. Nesse sentido, os elétrons, para a autora, seriam menos noções explicativas utilizadas pelos químicos do que instrumentos para agir sobre ou criar fenômenos, numa ação eminentemente operativa. A química antecipa a caracterização atual da ciência, assinalada por um realismo operativo, uma epistemologia do aprender fazendo, uma metaquímica orientadora da sua prática. Assim, não tem como valores fundamentais as verdades de suas construções, a explicação última da realidade, mas a possibilidade de suas representações (KOVAC, 2002; NORDMANN, 2006; BENSAUDE- VINCENT, 2009; RIBEIRO, 2014).

Para Nordmann (2006), orientado pela química, Bachelard é o primeiro a erigir o conceito de metaquímica: enquanto a metafísica se caracteriza por definir as condições de possibilidade da pesquisa científica, a metaquímica 
constituir-se-ia numa instância de orientação da prática científica; prática esta amplamente vinculada a uma relação dialética entre conceito e empiria, entre conceito e imagem, entre o signo e o significado (RIBEIRO, 2014). Nesse sentido, as práticas em química não são feitas para testar hipóteses teóricas (CALDIN, 1961). Concordando com Hoffmann (1993, 2007), Ribeiro, Silva e Bejarano (2014) entendem que os químicos não usam a mediação dos instrumentos para entender o fenômeno natural, como fazem os físicos, mas, concebendo a teoria como narrativas de experimentos, fazem história enquanto criam moléculas. Assim:

No tocante às teorias químicas, $\{\ldots\}$ diferentemente do que ocorre na física, que busca explicar fenômenos, recorrendo em sua grande parte ao princípio da causalidade, na química as teorias são narrativas e seus modelos e representações não pretendem falar necessariamente sobre o mundo, mas servir de ferramenta para intervir e transformar a natureza. Os modelos químicos não foram feitos para se referir ao real, mas para trabalhar com ícones representacionais que permitam intervir no real. Eles mostram o que é teoricamente possível e não o que é real. Dessa forma, uma síntese química não pode ser deduzida por um conjunto de leis. Átomos 
e moléculas são apenas - "atores no drama" da transformação química (RIBEIRO, 2014, p. 86).

Principalmente no contexto das análises e sínteses químicas, por vivenciar uma relação dialética e simbiótica entre ciência e tecnologia, entre empiria e representação, não é possível discernir a Química enquanto ciência e enquanto técnica. Assim, desenvolvendo o entendimento da Química como uma tecnociência, autores como Schummer (1997), Stokes (1997), Ziman (1984, 1999, 2000), Kovac (2001), Chamizo (2012) e Bensaude- Vincent (2009, 2010) consideram que a Química constitui-se em exemplo de conhecimento e prática que oscila entre as dimensões industrial, acadêmica, tecnológica e artística (RIBEIRO, 2014).

Uma tecnociência prioriza a realização de ações que buscam criar substâncias, entendendo que não existem substâncias sem uma ação criativa. Elas não são apenas o resultado de uma intencional ação humana, mas também uma construção de significados plenamente inseridos num dado contexto histórico-cultural. A principal forma de conhecimento dos químicos é propiciada por ações construtivas; e com estas, aumenta-se a complexidade do mundo. Desta maneira, a química deve ser entendida como uma tecnociência, uma tecnoquímica (CHAMIZO, 2012). Concordamos com Ribeiro (2014) que há uma forte relação 
entre a identidade química e os instrumentos de medida. A química, enquanto prática e enquanto conhecimento, somente será adequadamente compreendida do ponto de vista ontológico e epistemológico se inscrita em um contexto filosófico da filosofia da técnica, da tecnologia, dos instrumentos e do experimento: enquanto realismo operativo, enquanto fenomenotecnia, enquanto tecnoquímica (ROTHBART, 1993, 1999, 2002, 2007). Além disso, tal caráter antropicamente construtor desta ciência aliado ao dinamismo subjacente, pode, como frisa Hoffmann (2007), oferecer enormes contribuições não somente à filosofia da química, mas também à filosofia da ciência.

A fenomenologia química, para muitos filósofos da Química, deve ser compreendida no âmbito de uma dimensão bastante abrangente, considerando sua eminente pluralidade que se encontra para além do fato da ausência de unidade da teoria em que se baseia a conceitualização da química (LOMBARDI, 2012; LABARCA; LOMBARDI, 2005; RIBEIRO; COSTA-PEREIRA, 2012; RIBEIRO). “Falar de química como um campo unificado obscurece a pluralidade de métodos e tradições históricas e objetivos científicos deste campo, bem como as variedades de projetos interdisciplinares que os químicos trabalham" (SCHUMMER, 1998). 
No livro "O pluralismo coerente da química contemporânea" (BACHELARD, 2009), exclusivamente sobre filosofia química, traduzido para o português em 2009 e não muito usual mesmo para alguns estudiosos de Bachelard, o autor, não obstante o fato da maior parte dos filósofos não contemplarem a química em suas análises, estabelece reflexões sobre tal pluralismo da química. Este e a dimensão relacional constituem-se em dois aspectos selecionados pelo filósofo para caracterizar o que denominou como o racionalismo não lavoisieriano da química contemporânea. (BENSAUDE- VINCENT, 2008; RIBEIRO, 2014). Para Bachelard (1990, p. 74), "conhece-se claramente aquilo que se conhece grosseiramente. Se se pretende conhecer distintamente, o conhecimento pluraliza-se, o núcleo unitário do conceito explode.”

A Filosofia da Química entende a química como uma ciência fortemente inscrita em esquemas classificatórios, pensamentos diagramáticos, simbólicos, processuais, heurísticos, caracterizados pela razão prática, ao mesmo tempo em que, influenciada por valores estéticos, tem na criação, inovação e na dependência dos instrumentos e técnicas elementos que a complexificam intensamente (SILVA, 2015, p. 35). 
Segundo Ribeiro (2014), a química constitui-se em um campo científico marcadamente inscrito nas tensões entre ciência/técnica, academia/indústria, natural/artificial, útil/risco, além das grandes tensões filosóficas, micro/macro, parte/todo, contínuo/descontínuo, substância/processos, conceito/empiria, nomotético/ideográfico. Dessa forma, para Ribeiro, Silva e Bejarano (2014), esse pluralismo constitutivo (RIBEIRO; COSTA PEREIRA, 2012), ontológico (BACHELARD, 2009), metodológico (SCHUMMER, 1997, 2006), epistemológico (BACHELARD, 2009) e axiológico (KOVAC, 2002), perpassado por uma fenomenologia inscrita em complexas relações ontológicas, mobiliza simultaneamente: variados atores e contextos; variados estilos de pensamento; variados recursos cognitivos (HARRÉ, 2005; GILBERT, 2009; TALANQUER, 2005; HOFFMANN, 2003); variados valores pessoais e culturais envolvendo a categorização dos tipos naturais, relacionalidade, recursividade, lógica relacional e mereológica e uma relação constitutiva com os instrumentos de medida. Assim, entendemos que tais pluralismos que perpassam a Química constituem-se numa ampla fonte a ser explorada pela Filosofia da Ciência.

A característica interdisciplinar da investigação em química tem sido reiterada pela literatura (RIBEIRO, 2014; SCHUMMER, 2006). Nesse sentido, Sjöström (2006) salienta que os campos tradicionais da Química, como 
Analítica, Físico-química e Orgânica, foram alterados depois dos anos 1950: para este autor, ao longo das décadas de 1950 e de 1970, com a ocorrência dos respectivos processos de fisificação e bioficação, além de passar por uma revolução instrumental, a química adquire um caráter de ciência de serviço, transformando-se assim de ciência acadêmica em ciência pós-acadêmica e interdisciplinar.

De acordo com Schummer (2003), existem três tipos de noção de natureza em química: a estática, a teleológica e a dinâmica. A noção estática, associando a ideia de natural a uma essência original, derivada do ato de criação, considerava a arte como uma dimensão do artificial. A visão teleológica está associada à visão aristotélica do movimento princípio motor de cada ser em direção a um estado de perfeição. Ribeiro (2014), por sua vez, entende que do século XVIII emergiria uma concepção dinâmica de natureza, em que o natural e o artificial não mais estariam em contraposição.

Bachelard (1978), a partir de um entendimento do fenômeno natural como intrinsecamente mais pobre que o fenômeno artificial, considera que a construção fenomênica, realizada pelo químico em seus laboratórios, desenvolve-se numa espécie de esfera de proteção contra toda a perturbação irracional, que, para o filósofo, permeia o real natural. Em defesa intensa do caráter fenomenotécnico, 
aplicado, do real científico, o epistemólogo (1978, p.5) sublinha:

A realização de um programa racional de experiência determina uma realidade experimental sem irracionalidade. $\{\ldots\}$ o fenômeno ordenado é mais rico que o fenômeno natural. \{...\} O fenômeno realizado deve ser protegido contra toda a perturbação irracional. $\mathrm{O}$ racionalismo que nós defendemos fará assim face à polêmica que se apóia no irracionalismo insondável do fenômeno para afirmar uma realidade. Para o racionalismo científico, a aplicação não é uma derrota, um compromisso. Ele quer aplicar-se. Se se aplica mal, modifica-se. Não nega por isso seus princípios, dialetiza-os.

Nesse sentido, a natureza é também compreendida por Bachelard como um real que em si é pleno de irracionalidades. Daí, a necessidade premente do químico, em seu labor, construir realidades objetivamente racionais, eliminando irracionalidades que remetem ao natural dado, imerso em um processo de objetivação racional de um real. Um real que, mediante a aplicação simultaneamente técnica e racional adquire contornos noumênicos amplamente ricos. O químico, para Bachelard (1978, p.37) "só compreende a substância química quando constrói nela, 
através do pensamento, ligações íntimas. Mas já não se trata de uma construção de homo faber, somatório de gestos; trata-se sim, de uma construção coerente, limitada por numerosas interdições." Dessa forma, esse modo particular de conceber o lugar da natureza, entendida pelo químico mais como pretexto para a reificação de processos de objetivação do real, constitui-se numa emérita instância, estimuladora de eclosão de novas reflexões no bojo da Filosofia da Ciência.

\section{A FILOSOFIA DA QUÍMICA E A EDUCAÇÃO QUÍMICA: DIÁLOGOS, REFLEXÕES E POSSIBLIDADES}

Considerando as ligações entre a Filosofia da Química e a Educação Química, Labarca, Bejarano e Eichler (2013) entendem que reflexões sobre a natureza do conhecimento químico contribuem para a aprendizagem de conceitos químicos. Dessa forma, desejam que os avanços na investigação de diferentes e atuais tópicos na Filosofia da Química sejam utilizados em atividades de formação de professores a fim de que os docentes desenvolvam uma concepção epistemológica mais profunda da Química.

Ribeiro (2008), por sua vez, entende que as discussões em Filosofia da Química podem auxiliar os docentes na elucidação e clarificação de problemas básicos na Educação 
Química situados nas dimensões: ontológica, onde se inserem níveis de realidade, descrição, caracterização e instanciação dos entes químicos; epistemológica, a qual almeja a caracterização da natureza e especificidade da explicação química; e a heurísticometodológica, em que estão inseridas tanto as relações de superveniência, da ordem macromicro, como o essencial papel dos modelos para as interpretações químicas e as mediações didáticas. Para Ribeiro (2014, p. 35), “o debate da filosofia da química constitui um campo disciplinar com elementos intrínsecos em relação aos quais o currículo de química, e principalmente de formação de professores em química, deve tomar partido". Este autor problematiza o campo disciplinar da filosofia da química, defendendo a constituição do pluralismo da práxis química como um fundamento da educação química a partir de uma aproximação entre currículo, filosofia e química. Concordamos com Ribeiro (2014), a partir da análise temática da produção bibliográfica da filosofia da química, sobretudo aquela oferecida pelas revistas HYLE (International Journal for Philosophy of Chemistry) e Foundations of Chemistry, podem indicar um campo de problemas que podem orientar a didática da química, bem como o currículo, apesar de atualmente termos poucas aproximações entre filosofia e currículo (ERDURAN, 2001; ERDURAN; SCERRI, 2002). 
Todavia, é necessário ressaltar que, em grande parte, as discussões epistemológicas nos cursos de formação inicial e continuada de professores de Química são abordadas fundamentando-se em filósofos - como Kuhn, Lakatos, Popper e Feyerabend - cujas obras foram construídas tendo a Física como modelo de ciência natural. A Ciência Química impõe uma agenda de discussão no âmbito dos programas de formação inicial e continuada de docentes de Química que é própria da Filosofia da Química, o que inclui uma ampla variedade de eixos temáticos que vão desde o estatuto das leis e teorias químicas a tópicos metodológicos como experimentação, instrumentação e síntese química (LABARCA; BEJARANO; EICHLER, 2013). Mais ainda, entendemos que as problematizações acerca da especificidade da práxis química contemporânea e da própria relação entre a filosofia da química e a filosofia da ciência podem promover contribuições para a educação química, especificamente, para a formação de professores.

\section{NOTAS}

1 Doutorando em Ensino, História e Filosofia das Ciências (UFBA/UEFS). E-mail: professorlisandrobacelar@gmail.com. Agradecemos também ao Programa de Pós-Graduação em Ensino, 
Filosofia e História das Ciências e a Coordenação de Pessoal de Nível Superior (CAPES), pelo apoio.

2 Doutorando em Ensino, História e Filosofia das Ciências (UFBA/UEFS). E-mail: uarisonbarreto@gmail.com.

${ }^{3}$ Professor Associado do Departamento de Química Inorgânica e Geral da Universidade Federal da Bahia (UFBA). Doutor em Educação pela Universidade de São Paulo (Usp). E-mail: nelsonbejarano@gmail.com.

${ }^{4}$ Professor Adjunto pela Universidade Estadual do Sudoeste da Bahia (UESB). Doutor em Filosofia pela Universidade de Lisboa. E-mail: marcolimite@yahoo.com.br.

\section{REFERÊNCIAS BIBLIOGRÁFICAS}

ADÚRIZ-BRAVO, A. A. Integración de la epistemologia en la formación del professorado de ciencias. Tese de Doutorado Universitat Atonoma de Barcelona, Barcelona, 2001.

BACHELARD, G. A filosofia do não. São Paulo: Abril Cultural Ospensadores, 1978a. (Originalmente publicado em 1940).

BACHELARD, G. O novo espírito cientifico. São Paulo: Abril Cultural Os pensadores, 1978b. (Originalmente publicado em 1934).

BACHELARD, G. Thing knowledge: A Philosophy of Scientific Instruments. University of Los Angeles; London: California Press, 2004. 
BACHELARD, G. O pluralismo coerente da química moderna. Rio de Janeiro: Contraponto, 2009.

BENFEY, T. Reflections on the Philosophy of Chemistry and a Rallying Call for Our Discipline. Foundations of Chemistry, New York, v.2, p.195-205, 2000.

BENSAUDE-VINCENT, B. Chemistry in the french tradition of philosophy of science: duhem, meyerson, metzger and bachelard. Studies in history and philosophy of science, [S.I], v.36, n.4, 2005, p.627- 848 .

BENSAUDE-VINCENT, B. Matire penser, essais d'histoire et de philosophie de la chimie. Paris:Presses de l'Universit Paris Ouest, 2008.

BENSAUDE-VINCENT, B. The chemists' style of thinking. Ber.wissenschaftsgesch, [S.1], n.32, 2009, p.365-378.

BENSAUDE-VINCENT, B; NUROCK, V, "Ethique des nanotechnologies," in Traité de bioéthique, ed. Emmanuel Hirsch, vol. 1, 355-369 (Paris, Érès, 2010).

BOGDAN, R. S.; BIKEN, S. Investigação qualitativa em educação: uma introdução à teoria e aos métodos. 12.ed. Porto: Porto, 2003.

CALDIN, E. F. The structure of Chemistry in relation to the philosophy of science. London; New York: Sheed e Wards, 1961.

CHAMIZO, J (2012). A. Technochemistry. One of the chemists' ways of knowing (prelo). 
COSTA PEREIRA, D. Epistemologia da química e estrutura e lógica dos seus discursos, química. Lisboa: SPQ.

HARRÉ, R. (2005). Chemical kindsand essences revisited. Foundations of Chemistry. New York, v.7, 1995, p. 7-30.

HOFFMANN, J. M.L. How Should Chemists Think? Scientific American, 1993, p.66-73.

HOFFMANN, J. M.L. Thoughts on aesthetics and visualization in chemistry. HYLE. International Journal for Philosophy of Chemistry, Berlin, v.9, 2003, p.7-10.

HOFFMANN, J. M.L. O mesmo e o não-mesmo. São Paulo: Unesp. HYLE - International Journal for Philosophy of Chemistry 9, 2007, pp. 7-1.

IZIQUIERDO-AYMERICH, M.; ADÚRIZ-BRAVO, A. Epistemological Foundations of school science. Science \& education, New York, v.12, n.1, 2003, p. 27-43.

IZIQUIERDO - AYMERICH, M. La ensenanza de los componentes prácticos y axiológicos de lós conceptos químicos. In: CABRÉ, M. T.; BACH, C. (eds.), Coneixement, llenguatge $I$ discurs especialitztat. Barcelona: Institut Universitari de Linguistica Aplicada (UFP)/Documenta Universitária, 2012.

KOVAC, J. Gifts and commodities in chemistry. HYLE International Journal for Philosophy of Chemistry, Berlin, v.7, n.2, 2001, p.141-153.

KOVAC, J. Theoretical and practical reasoning in chemistry. Foundations of Chemistry. New York, v. 4, 2002, p. 63-171. 
GILBERT, J. K. Visualization: a metacognitive skill. In science and science education. In: - Visualization in Science Education. Holanda: Springe, 2009.

HENDRY, R. F. "Molecular Models and the Question of Physicalism", HYLE - International Journal for Philosophy of Chemistry 5, 1999, pp. 143-60.

LABARCA, M. La filosofia de la química en la filosofia de la ciência contemporanea. Redes, [S.l], v.11, n. 021, 2005, p.155171.

LABARCA, M.; LOMBARDI, O. The ontological autonomy of the chemical world. Foundations of Chemistry. 2005, v. 7, n. 2, p. 125-148.

LABARCA, M.; BEJARANO, N.R.R.; EICHLER, M. L. Química e filosofia: rumo a uma frutífera colaboração. Química Nova, v. 36, n. 8, 2013, p.1-17.

LEWIS, G. N. (1923). Valence and the Structure of Atoms and Molecules. New York: Chemical Catalog Co. Reprinted, New York: Dover, 1966.

LOMBARDI, O. Prigogine and the Many Voices of Nature. Foundations of Chemistry, Vol. 14, n. 3, 2012, pp. 205-219.

NORDMANN, A. From metaphysics to metachemistry. In: BAIRD, Davis; SCERRI, Eric; MCINTYRE, Lee (eds.). Philosophy of Chemistry: synthesis of a new discipline. Boston Studies in the Philosophy of Science, Dordrecht: Springer, 2006.

RIBEIRO, M. A. P.; SILVA, L. B.; BEJARANO, N. R. R. Os clássicos da filosofia no contexto da química. In: XVII 
Encontro Nacional de Ensino de Química, 2014, Ouro Preto. XVII Encontro Nacional de Ensino de Química, 2014.

RIBEIRO, M. A. P. Filosofia e Química: Miscíveis- Quais as implicações da Filosofia da Química para o Ensino Médio? XIV ENEQ, 2008.

RIBEIRO, M.A.P. Integração da Filosofia da química no currículo de formação inicial de professores. Contributos para uma filosofia no ensino. Tese doutoral. Universidade de Lisboa: Lisboa, 2014.

ROTHBART, D. D. Discovering Natural Kinds Through Inter-Theoretic Prototypes. Methodology and Science, [S.1], v.26, 1993, p.171-189.

ROTHBART, D. D. Spectrometers as Analogues of Nature. Philosophy of Science. Association (PSA), [S.1], v.1, 1994, p.141-148.

ROTHBART, D. D.; S.W. SLAYDEN. The Epistemology of a Spectrometer. Philosophy of Science, Chicago,v.61, 1994, p.2538.

ROTHBART, D. D. The dual nature of chemical substance. Techné, [S.1], v.6, n.2, 2002, p.106-109.

ROTHBART, D. D. Philosophical Instruments: Minds and Tools at Work. Urbana: University of Illinois, 2007.

SCERRI, E.R. The Ambiguity of Reduction, HYLE International Journal for Philosophy of Chemistry 13, 2007, pp. 67-81. 
SCERRI, E.R. Philosophy of chemistry: A new interdisciplinary field? Journal of Chemical Education, v. 77, n. 4 , p. 522-525, 2000 b.

SCERRI, E.R. Normative and descriptive philosophy of science and the role of Chemistry. In: DAVIS BAIRD, Scerri, Eric; MCINTYRE, lee (eds.). Philosophy of Chemistry: synthesis of a new discipline. Boston studies in the philosophy of science, v.242, 2006, p.119-128.

SCERRI, E.R. The Periodic Table: Its Story and Its Significance. New York: Oxford University Press, 2007.

SCHUMMER, J. Towards a philosophy of Chemistry. Journal for General Philosophy of Science,[S.1], v. 28, 1997, p.307-335.

SCHUMMER, J. Scientometric studies on chemistry I: the exponential growth of Chemical substances, 1800-1995. Scientometrics,[S.1], v. 39, 1997a, p. 107-123.

SCHUMMER, J. Scientometric studies on chemistry ii: aims and methods of producing New chemical substances.Scientometrics, [S.1],v.39, 1997b, p.125-140.

SCHUMMER, J. The philosophy of Chemistry: From infancy towards maturity. In: BAIRD, Davis; SCERRI, Eric; MACINTYLEE, Lee (eds.). Philosophy of Chemistry: Synthesis of a new Discipline. Dordrecht: Springer, p. 19-39.

SCHUMMER, J. The Notion of Nature in Chemistry. Studies in History and Philosophy of Science, v.34, 2003a, p. 705-736.

SCHUMMER, J. Editorial: Substances versus Reactions. HYLE- International Journal for Philosophy of Chemistry, Berlin, v.10, n.1, 2004. 
SCHUMMER, J. The Preference of Models over Laws of Nature. HYLE - International Journal for Philosophy of Chemistryin Chemistry. In: European Review, v.22, n.1, 2014, p. 87-101.

SECO, M.; ALVAREZ, S.; SALES, J. On books and chemical elements. Foundations of Chemistry. New York, v.10, n.2, 2008, p.79-100.

SILVA, L.B. Concepções de fenômeno químico de docentes formadores e pós-graduandos das áreas de Química e ensino de Química (Dissertação de Mestrado) - Universidade Federal da Bahia, Universidade Estadual de Feira de Santana (UFBA/UEFS), Salvador, 2015.

SILVA, L. B.; BEJARANO, N. R. R.; RIBEIRO, M. A. P. Bachelard: an authentic philosopher of Chemistry. In: International Society For The Philosophy Of Chemistry. 2830/07/2015. Rio De Janeiro. Anais. Rio de Janeiro, 2015.

SJÖNSTRÖM, J. Beyond classical chemistry: Subfields and metafields of the molecular sciences. Chemistry International, 28(September-October), 2006, p. 9-15.

SJOSTROM, J. "The Discourse of Chemistry (and Beyond)", HYLE - International Journal for Philosophy of Chemistry 13, 2007, pp. 83-97.

STOKES, D. E. Pasteur's quadrant. Washington: Brookings institution press. Scientometrics, v.39, 1997, p.125-140.

TALANQUER, V. El Químico Intuitivo. Educación Química, México, v.16, n.4, 2005, p. 114-122. 
VAN BRAKEL, J. On the neglect of the philosophy of Chemistry. Foundations of Chemistry. New York, v.1, 1999, p.111-174.

VAN BRAKEL, J. Philosophy of Chemistry: Between the manifest and the scientific image. Leuven: Leuven University Press, 2000.

VAN BRAKEL, J. Kant's legacy for the philosophy of Chemistry. In: BAIRD, D., et al. (Eds.) Philosophy of Chemistry, Springer: The Netherlands, 2006.

VAN BRAKEL, J. On Pré-history of philosophy of Chemistry. In: International Society For The Philosophy Of Chemistry Summer Symposium, 2012, Leuven, Bélgica.

VIHALEMM, R. Are Laws of Nature and Scientific Theories Peculiar in Chemistry? Scrutinizing Mendeleev's Discovery. Foundations of Chemistry. New York, v.5, 2003, p.7-22.

VIHALEMM, R. The autonomy ofChemistry: old and new problems. Foundations of Chemistry. New York, v.13, n.2, 2011, p.97-107.

ZIMAN, J. An introduction to science studies - the philosophical and social aspects of science and technology. Cambridge: Cambridge University Press, 1984.

ZIMAN, J. A ciência na sociedade moderna. In: GIL, F. (Coord.). A ciência tal qual se faz. Lisboa: Edições João Sá da Costa, 1999, p. 437-450.

ZIMAN, J. Real science - what is, and what it means. Cambridge: Cambridge University Press, 2000. 